\title{
Preventive effects of sea cucumber (Apostichopus japonicus) ethanol extract on palmitate-induced vascular injury in vivo
}

\author{
Chunying Zhang ${ }^{\dagger}$, Seon-Heui Cha* \\ Department of Marine Bio and Medical Sciences, Hanseo University, Seosan 31962, Korea
}

\begin{abstract}
Cardiovascular diseases (CVDs) have posed serious public health problems, accounting for nearly 30\% of mortality worldwide and their incidence is still increasing. Therefore, new treatment resources are necessary to prevent or manage the ever-increasing population of patients with CVDs. Sea cucumber is well known for its medical and health benefit effects, but it is not well known what/how effect it has on vascular disease. In the present study, we examined the protect effect of sea cucumber, Apostichopus japonicus $80 \%$ ethanol extract (AJE) on zebrafish embryo with the stimulation of free fatty acid, palmitate (PA). In vivo study showed that AJE can attenuate PA-induced toxicity through relieving the rapid heartbeat, increasing the survival rate and reducing the malformation in both wild type and $\mathrm{Tg}$ (fli1a:eGFP) transgenic zebrafish lines. Additionally, compare with PA treated embryos, the yolk sac area, body length, axial vascular segment (AVS) and intersegmental vessel (ISV) of the co-treatment group of AJE and PA were comparable to the control group. Moreover, AJE lowered the expression of inducible nitric oxide synthase (iNOS), nitric oxide (NO) and inflammation-related genes induced by PA, and inhibited PA-induced vascular development disorders. Our data preliminarily verify that AJE could be a candidate resource for the prevention or therapy of CVDs.
\end{abstract}

Keywords: Apostichopus japonicus, Marine natural product, Palmitate, Vascular, Inflammation

\section{Introduction}

Cardiovascular diseases (CVDs) have gained more attention due to their high morbidity and mortality worldwide (Kaptoge et al., 2019). CVD is a multifactorial disease, which could be caused by inflammation, hypertension, atherosclerosis, dyslipidemia, insulin resistance, elevated heart rate and enteric dys- bacteriosis (Ebbesson et al., 2015; Zhou et al., 2021). Endothelial cells play an essential role in cardiovascular homeostasis which are important constituents of blood vessels. Normal vascular endothelium is considered as a gatekeeper of cardiovascular health, while abnormal vascular endothelium is the main cause of CVDs (Sun et al., 2020). Extensive evidence supports that inflammation plays a key role in the pathogenesis of CVDs by

Received: Jan 13, 2022 Revised: Jan 26, 2022 Accepted: Jan 31, 2022

${ }^{\dagger}$ The authors current affiliation: College of Agronomy, Jilin Agricultural University, Changchun 130118, China

${ }^{*}$ Corresponding author: Seon-Heui Cha

Department of Marine Bio and Medical Sciences, Hanseo University, Seosan 31962, Korea

Tel: +82-41-660-1550, E-mail: sunnyday8109@gmail.com

This is an Open Access article distributed under the terms of the Creative Commons Attribution Non-Commercial License (http://creativecommons.org/licenses/by$\mathrm{nc} / 4.0 /$ ) which permits unrestricted non-commercial use, distribution, and reproduction in any medium, provided the original work is properly cited.

Copyright $\odot 2022$ The Korean Society of Fisheries and Aquatic Science 
promoting endothelial cell activation or dysfunction (Carter, 2012). Therefore, the prevention, systematic management and effective intervention of vascular disease are particularly important and necessary (Liu and Wang, 2016).

Saturated fatty acids (FAs) such as palmitate (PA) have been associated with cardiovascular mortality, which was thought to induce chronic low-grade inflammation, activate the innate immune system, and bring harmful effects on vascular cells and other tissues and organs through the inflammatory processes (Ebbesson et al., 2015; Volpe et al., 2014). Vascular dysfunction is tumor necrosis factor- (TNF-) $\alpha$ dependent and is related to the increase of inducible nitric oxide synthase (iNOS) levels in aortic endothelial cells and excessive generation of nitric oxide (NO), which is the reason for the reduced reactivity of aortas to vasoconstrictors. Therefore, TNF- $\alpha$ blockers might be useful in the prevention of CVDs (Aires et al., 2013) . In addition, LIM domain only 2 ( $\mathrm{Lmo} 2$ ) is an essential gene for vertebrate primitive hematopoiesis and normal endothelial development (Zhu et al., 2005); Friend leukemia integration 1 transcription factor (Fli1) plays an important physiological role in vascular development at the top of the transcriptional network that regulates blood and endothelial development (Li et al., 2015). Thus, maintaining the balanced expression of vascular-related genes also plays a positive role in preventing the occurrence of CVDs (Seo et al., 2006).

At present, several synthetic drugs have been applied for treatment of CVDs, whereas some adverse effects such as arrhythmias, hyperkalemia and gastrointestinal reaction are giving cause for concern (Giudicessi et al., 2018; Ziff and Kotecha, 2016). On the contrary, mounting evidence suggests that natural products could be a safe and effective alternative for the prevention and treatment of CVDs (Miller et al., 2017; Tang et al., 2017; Tufail et al., 2018). Sea cucumber, belongs to the class of Holothuroidea and the phylum of Echinodermata, has attracted much attention as a marine functional food due to its biological activity and medicinal properties (Hossain et al., 2020). Several studies have confirmed that sea cucumbers and their extracts have various potential containing anti-inflammatory (Kareh et al., 2018), antithrombotic (Chen et al., 2012), anticoagulant (Mansour et al., 2019), hypoglycemic activities (Hu et al., 2013) and hypolipidemic (Liu et al., 2002), all of which have significant impact on the prevention and treatment of CVDs.

Apostichopus japonicus (A. japonicus) is a species of sea cucumber in the family Stichopodidae. Previous studies have shown that $A$. japonicus polysaccharides have antioxidant and antihyperlipidemic activities (Liu et al., 2012). Moreover, the nortriterpene and triterpene glycosides from A. japonicus displayed antifungal activities (Wang et al., 2012). And the depolymerized glycosaminoglycan from A. japonicus showed anticoagulant activities (Yang et al., 2015a; Yang et al., 2015b). Furthermore, A. japonicus extract (AJE) has been shown to have anti-skin cancer activities (Kim et al., 2017). However, the research evidence about the function of $A$. japonicus in vascular dysfunction has not been revealed. Zebrafish is recognized as a link between invertebrate and mammalian models due to its vascular development of the circulatory system and molecular pathways are highly similar with human and other higher vertebrates (Li et al., 2014). In recent years, the vascular-specific $\mathrm{Tg}$ (fli1a:eGFP) transgenic zebrafish has been exceptionally useful for examining vascular development in zebrafish (Lawson and Weinstein, 2002). Therefore, the present study applied PA to stimulate human umbilical vein endothelial cells (HUVECs) and zebrafish embryos to establish in vivo and in vitro models to evaluate whether the extracts from A. japonicu have protective effect against PA-induced vascular damage.

\section{Materials and Methods}

\section{Natural product extract and zebrafish}

A $80 \%$ of ethanol extract of A. japonicus (AJE) was donated from Marine Bio Resource Information System (MBRIS), https://www.mbris.kr/.

The embryos of wild type zebrafish were derived from the zebrafish system of our lab. The management of zebrafish was in accordance with established procedures, http://zfin.org/zf_info/ zfbook/zfbk.html. Transgenic zebrafish line $\mathrm{Tg}$ (flila:eGFP) was acquired from Zebrafish Center for Disease Modeling (ZCDM). Zebrafish embryos used in the experiment were processed according to the guidelines of Hanseo University.

\section{Palmitic acid preparation}

Palmitic acid (PA; Sigma-Aldrich, St. Louis, MO, USA) was dissolved in pre-heated $0.1 \mathrm{~N} \mathrm{NaOH}$ (Daejung, Korea) at $70^{\circ} \mathrm{C}$ and conjugating with FA-free bovine serum albumin (BSA; Sigma-Aldrich) to obtain a $10 \mathrm{mM}$ stock solution, which was improved based on the previous report (Sinha et al., 2004). We prepared two stock solutions: one for embryos, which mixing $20 \mathrm{mM}$ PA with 12\% BSA (w/v) containing DPBS (Dulbecco's Phosphate Buffered Saline, Welgene) at a ratio of 1:1; another one for cell culture that mixing $20 \mathrm{mM}$ PA with $12 \%$ BSA (w/ 
v) containing DMEM (Dulbecco's Modified Eagle's Medium, Welgene, Gyeongsan, Korea) at a ratio of 1:1. Control solution contained $\mathrm{NaOH}$ and BSA without lipids.

\section{Treatment of zebrafish embryos with A. japonicus extract} (AJE) and palmitate (PA) and phenotypic analysis

$\mathrm{Tg}$ (fli1a:eGFP) transgenic embryos were applied to evaluate the effect of AJE on vascular formation under PA treatment. Embryos at 24 hours post-fertilization (hpf) were distributed to 12-well plate (20 embryos/well) and incubated in the AJE $(25 \mu \mathrm{g} / \mathrm{mL})$ for $1 \mathrm{~h}$ prior to the additional PA $(2 \mathrm{mM})$ in $2 \mathrm{~mL}$ embryo media $(0.003 \%$ sea salt, $0.0075 \%$ calcium sulfate) including $75 \mu \mathrm{M}$ phenylthiourea (PTU, Sigma-Aldrich) for $96 \mathrm{hpf}$ at $28.5^{\circ} \mathrm{C}$. The phenotype of embryos was checked daily, the malformation and mortality were calculated, and the dead embryos were removed from the well in time. The phenotype morphology, yolk sac area and body length of embryos were observed by a stereomicroscope (SZ61, Olympus, Tokyo, Japan) and measured using cellSens software (Olympus). Vascular morphology, axial vascular segment (AVS) and intersegmental vessel (ISV) were observed and measured by a fluorescent microscope (SZX16, Olympus).

\section{Heartbeat measurement}

Heart rate is an important indicator reflecting the toxicity of cardiac development (Sarmah and Marrs, 2016). After embryos were exposed to PA or AJE/PA from 24 to $75 \mathrm{hpf}, 12$ embryos were randomly selected from each group and the heart rate was recorded by counting the beats per minute under stereomicroscope (SZ61). The results were presented in average heart rate per minute.

\section{Determination of nitro oxide (NO) production and apoptotic cells in zebrafish}

NO production was measured in living zebrafish embryos by a fluorescent probe dye of diaminofluorophore 4-amino-5-methylamino-2',7'-difluorofluorescein diacetate (DAF-FM DA, Invitrogen, Carlsbad, CA, USA). In the presence of dioxygen, NO transforms DAF-FM DA to produce highly fluorescent triazole derivatives. Cell death was determined in living zebrafish embryos by staining with acridine orange (AO, Sigma-Aldrich). $\mathrm{AO}$ is a kind of cell-permeable nucleic acid dye that can be released into the nucleus when a cell begins to die, and interacts with partially uncoiled DNA to produce high fluorescence (Thomé et al., 2016). The embryos developed to $86 \mathrm{hpf}$ were rinsed and stained with $5 \mu \mathrm{M}$ DAF-FM DA or $5 \mu \mathrm{g} / \mathrm{mL}$ AO for
$30 \mathrm{~min}$ at $28.5^{\circ} \mathrm{C}$ in the dark. After fully rinsed and anesthetized by $0.003 \%$ methanesulfonate (MS-222), the embryos were observed and photographed under a fluorescent microscope (SZX16, Olympus). The fluorescence intensity of images was quantified by ImageJ software (http://rsb.info.nih.gov/ij) and the triplicate mean value were represented by a bar graph.

\section{RNA extraction and real time quantitative PCR (qPCR) analysis}

Total RNA was isolated from zebrafish embryos using TRIzol Reagent (Invitrogen), and RNA was reverse-transcribed to cDNA by cDNA synthesis PrimeScript ${ }^{\mathrm{TM}} 1$ st strand cDNA Synthesis kit (Takara) following the manufacturer's instructions. qPCR was performed using TOPreal ${ }^{\mathrm{TM}}$ qPCR 2X PreMIX (SYBR Green with low ROX, Enzynomics) on Qiagen Rotor-Gene Q instrument (Hilden Düsseldorf, Germany). Gene expression levels were acquired based on the normalization of the endogenous $\beta$-actin gene, which was not affected by PA. Relative expression was calculated with $\Delta \Delta \mathrm{CT}$ method. The sequences of q-PCR primer pairs were presented in Table 1.

\section{Statistical analysis}

All graphs were generated by GraphPad prim version 9.0 (GraphPad software, San Diego, CA, USA) and one-way analysis of variance (ANOVA) with subsequent multiple comparison test (Tukey) were applied for significance analysis. Data are presented as mean \pm SD. $p<0.05$ was considered statistically significant.

Table 1. Primer sequences

\begin{tabular}{|c|c|c|c|}
\hline $\begin{array}{l}\text { Gene } \\
\text { name }\end{array}$ & & Sequence $5^{\prime}-3^{\prime}$ & Gene ID \\
\hline$I L-1 \beta$ & $\begin{array}{l}\text { Forward } \\
\text { Reverse }\end{array}$ & $\begin{array}{l}\text { TCAAACCCCAATCCACAGAG } \\
\text { TCACTTCACGCTCTTGGATG }\end{array}$ & 405770 \\
\hline TNF- $a$ & $\begin{array}{l}\text { Forward } \\
\text { Reverse }\end{array}$ & $\begin{array}{l}\text { AGAAGGAGAGTTGCCTTTACCGCT } \\
\text { AACACCCTCCATACACCCGACTTT }\end{array}$ & AB183467.1 \\
\hline$i N O S 2 b$ & $\begin{array}{l}\text { Forward } \\
\text { Reverse }\end{array}$ & $\begin{array}{l}\text { GTTTGAAAGGCAATCCGATGA } \\
\text { GCTGTTGTGATGCTGCTTAGAGT }\end{array}$ & XM_017350981.2 \\
\hline NF-KB p65 & $\begin{array}{l}\text { Forward } \\
\text { Reverse }\end{array}$ & $\begin{array}{l}\text { TCCCTGGAGAGAAGAGCAAC } \\
\text { CAGTCTTTTCCCACCAGCTC }\end{array}$ & 415099 \\
\hline Fli1 & $\begin{array}{l}\text { Forward } \\
\text { Reverse }\end{array}$ & $\begin{array}{l}\text { ATGCGTCTTATGATGCTGTACG } \\
\text { TTGGTTCCTTCCCAGGTGAT }\end{array}$ & 30619 \\
\hline Lmo2 & $\begin{array}{l}\text { Forward } \\
\text { Reverse }\end{array}$ & $\begin{array}{l}\text { GTTTTGTGCGGCAGATGGT } \\
\text { GCCTTCAGAAAGAAGCGGTC }\end{array}$ & NM_131111.1 \\
\hline$\beta$-actin & $\begin{array}{l}\text { Forward } \\
\text { Reverse }\end{array}$ & $\begin{array}{l}\text { AATCTTGCGGTATCCACGAGACCA } \\
\text { TCTCCTTCTGCATCCTGTCAGCAA }\end{array}$ & AF057040.1 \\
\hline
\end{tabular}

IL-1, interleukin 1; TNF, tumor necrosis factor; iNOS, inducible nitric oxide synthase; NF-KB, nuclear factor kappa-light-chain-enhancer of activated B; Fli, friend leukemia integration 1 transcription factor: Lmo, LIM domain only. 


\section{Results}

Protective effects of $A$. japonicus extract (AJE) against palmitate (PA)-induced embryotoxicity in zebrafish

To evaluate the toxicity of PA on embryonic development in zebrafish, we investigated the survival rate of embryos treated with PA (1.5-3 mM) at the $24 \mathrm{hpf}$ stage during $62 \mathrm{~h}$ and screened the concentration of PA. As shown in Table 2, $20 \%$ and $100 \%$ lethality occurred by $50 \mathrm{hpf}$ at $2.5 \mathrm{mM}$ and $3 \mathrm{mM}$ of PA, respectively. No mortality occurred in embryos at 1.5 and 2 $\mathrm{mM}$ by $86 \mathrm{hpf}$ and we selected a concentration of $2 \mathrm{mM}$ PA for subsequent experiments. Furthermore, we confirmed that AJE alone had no toxicity to embryos in the concentration range of $5-50 \mu \mathrm{g} / \mathrm{mL}$ since no significant change in heart rate (Table 3 ).

To determine whether AJE has a protective effect on PA-induced embryotoxicity, we analyzed the developmental phenotypes containing heartbeat, survival, malformation, yolk sac area and body length, to identify morphological differences of $24 \mathrm{hpf}$ embryos after exposed to PA (2 mM PA) alone or 1 h pretreatment with AJE $(25 \mu \mathrm{g} / \mathrm{mL})$ prior to PA. The results showed pretreatment with AJE could reduce the PA-induced acceleration of heartbeat at $75 \mathrm{hpf}$ and increase embryos survival rate at 55 and $75 \mathrm{hpf}$ relative to the control group (Fig. 1a, b). Meanwhile, PA treated embryos revealed morphological abnormalities, including spinal curvature, edema, and delayed absorption of yolk sac, which were alleviated by pretreatment

Table 2. Phytotoxicity evaluation of different concentrations of AJE on zebrafish embryos

\begin{tabular}{llllll}
\hline Treatment at 7 hpf & \multicolumn{6}{l}{ AJE $(\mu \mathrm{g} / \mathrm{mL})$ in egg water } \\
\cline { 2 - 6 } & 0 & 5 & 10 & 50 & 100 \\
\hline Mortality (50 hpf) & $0 / 20$ & $0 / 20$ & $0 / 20$ & $0 / 20$ & $0 / 20$ \\
Heartbeat (3 dpf) & $180 \pm 15$ & $171 \pm 14$ & $186 \pm 19$ & $186 \pm 19$ & $129 \pm 9^{*}$ \\
\hline
\end{tabular}

Each group measured 12-18 embryos.

Significantly different $(p<0.05)$ compare with control $(0 \mu \mathrm{g} / \mathrm{mL})$.

hpf, hours post fertilization; AJE, Apostichopus japonicus extract; dpf, days post fertilization.

Table 3. Toxicity evaluation of different concentrations of PA on zebrafish embryos

\begin{tabular}{llllll}
\hline Treatment at 24 hpf & \multicolumn{6}{l}{ PA (mM) in egg water with PTU } \\
\cline { 2 - 6 } & 0 & 1.5 & 2 & 2.5 & 3 \\
\hline Mortality (50 hpf) & $0 / 20$ & $0 / 20$ & $0 / 20$ & $4 / 20$ & $20 / 20$ \\
Heartbeat (2 dpf) & $145 \pm 5$ & $150 \pm 5$ & $159 \pm 14^{* *}$ & - & - \\
\hline
\end{tabular}

"Significantly different $(p<0.01)$ compare with control $(0 \mathrm{mM})$.

$\mathrm{PA}$, palmitate; $\mathrm{hpf}$, hours post fertilization; PTU, phenylthiourea; $\mathrm{dpf}$, days post fertilization. with AJE (Fig. 1c, d). In addition, the yolk sac area and body length were larger and shorter than that of the control group, respectively, which were also improved by AJE treatment (Fig. $1 e, f)$.

Inhibitory effect of AJE on PA-induced NO generation, inflammatory cytokine mRNA expression and cell death in zebrafish embryos

The overproduction of NO in blood vessels is related to circulatory failure, which occurs in systemic inflammatory response and may have cytotoxicity to surrounding tissues (Stoclet et al., 1998). Therefore, we examined whether excessive NO induced by PA ( $2 \mathrm{mM})$ could be reduced by AJE in zebrafish. After pretreatment with AJE $(25 \mu \mathrm{g} / \mathrm{mL})$, the excessive NO production induced by PA was significantly reduced compare with control (Fig. 2a). Moreover, mRNA levels of inflammatory cytokines such as iNOS and TNF- $\alpha$ were enhanced by PA, while significantly decreased by AJE (Fig. 2b, c). TNF- $\alpha$ has been shown to induce NF- $\kappa \mathrm{B}$ activation which result in cell death (Zhi et al., 2015). Our results showed that PA-induced NF- $k B$ activation which was attenuated by AJE (Fig. 2d). Meanwhile, PA-induced cell death was significantly higher than in the control group, however, pretreatment AJE significantly reduced the level (Fig. 2e). These results suggest that AJE can protect zebrafish embryos against PA-induced inflammation and cell death.

\section{A. japonicus extract (AJE) improves the effect of palmitate (PA) on vascular morphogenesis and relative genes expres- sion in Tg (fli1a:eGFP) transgenic zebrafish}

Vascular morphogenesis includes three major processes that vasculogenesis, angiogenesis and vascular remodeling (Patan, 2000), among which angiogenesis plays a key role in the pathological process of CVDs (Zhang et al., 2018). To evaluate the phenotypic effects of PA or AJE treatment on vascular morphogenesis, we measured the length of AVSs and ISVs in $T g$ (fli1a:eGFP) transgenic zebrafish embryos treated with $2 \mathrm{mM}$ $\mathrm{PA}$, or co-treated with AJE at $24 \mathrm{hpf}$. The results showed the length of AVSs and ISVs are both significantly decreased after PA treatment compare with control at $60 \mathrm{hpf}$. However, it has been improved by pretreatment of AJE (Fig. 3a, b). In addition, we determined the mRNA levels of Fli1 gene, which regulates endothelial development, as well as Lmo2, a key gene necessary for angiogenesis and related to ISV formation (Ganta and Annex, 2017; Li et al., 2015; Meng et al., 2016). The results showed that the mRNA expression of both Lmo2 and Fli1 in PA-treated 
a

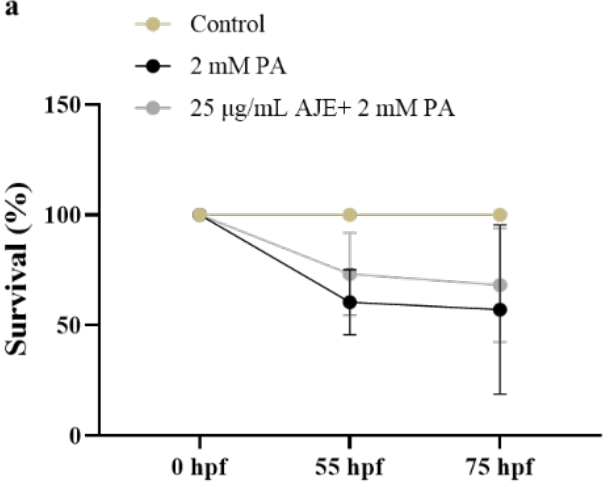

b

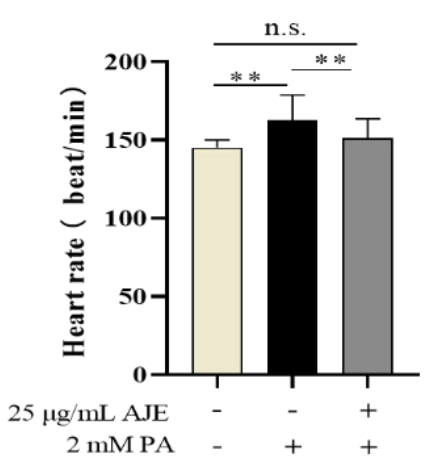

c

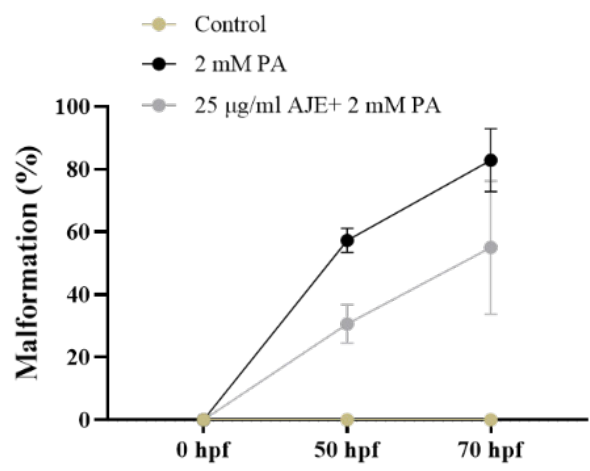

d

Control

2 mM PA

$25 \mu \mathrm{g} / \mathrm{mL}$ AJE $+2 \mathrm{mM}$ PA
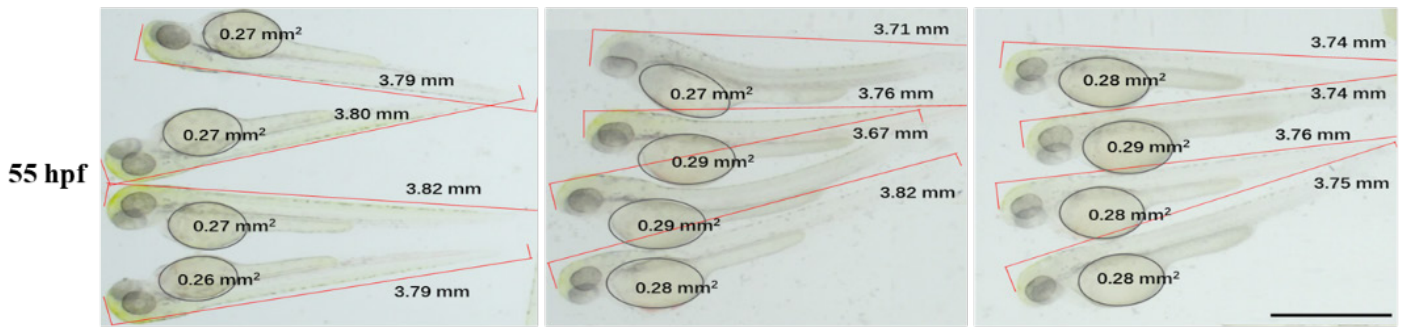

e

\section{f}
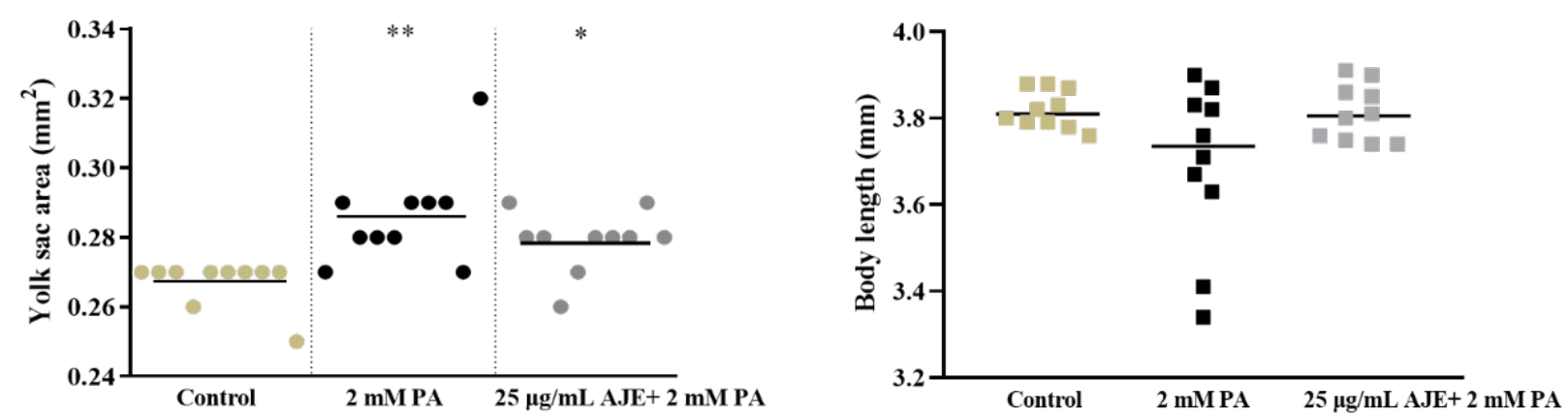

Fig. 1. Apostichopus japonicus extract (AJE) attenuates palmitate (PA)-induced toxicity in zebrafish embryos. Embryos were treated with $2 \mathrm{mM} \mathrm{PA}$, or co-treated with $25 \mu \mathrm{g} / \mathrm{mL}$ AJE in egg water with $75 \mu \mathrm{M}$ phenylthiourea (PTU) at 24-25 hpf. (a) Heart rates of zebrafish were measured at $75 \mathrm{hpf}$, the results are expressed as the beats/min. (b) survival rate of zebrafish were measured at $55 \mathrm{hpf}$ and 75 hpf. (c) malformation were measured at $50 \mathrm{hpf}$ and $70 \mathrm{hpf}$. (d) morphology was photographed at 55 hpf. (e) yolk sac area (mm ${ }^{2}$ ) and $(\mathrm{f})$ body length (mm) of zebrafish were measured at 55 hpf. $\mathrm{n}=10-50$. Scale bar: $1 \mathrm{~mm} .{ }^{*} p<0.05$ and ${ }^{* *} p<0.01$. hpf, hours post fertilization.

groups was significantly higher than those in control group, while the expression level of the AJE pretreatment group was reduced to a level comparable to that of the control group (Fig. $3 c, d)$.

\section{Discussion}

The etiology of CVDs is multifactorial, lifestyle changes and drug treatment can reduce the incidence of CVDs, such as heart 
a
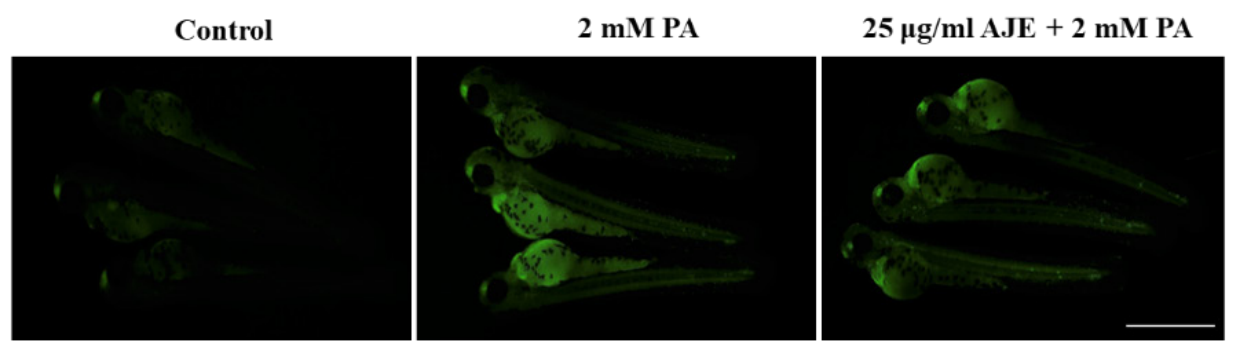

c

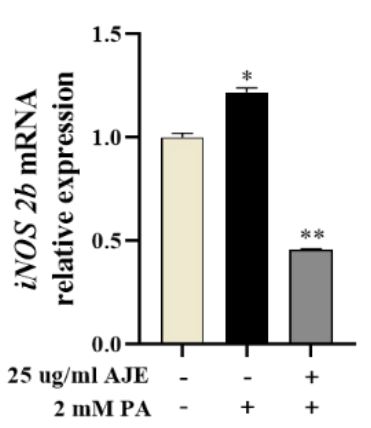

e

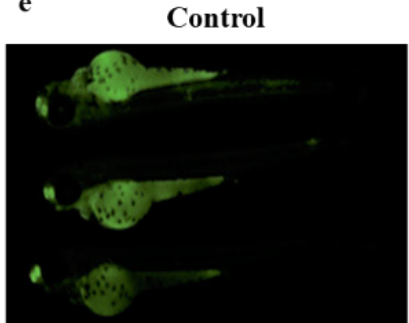

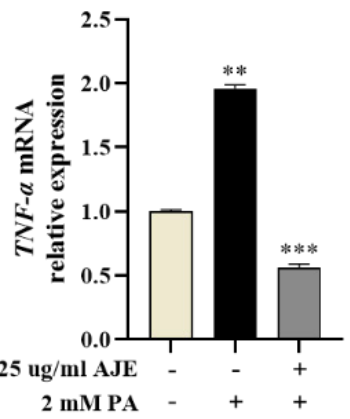

2 mM PA

$25 \mu \mathrm{g} / \mathrm{ml} \mathrm{AJE}+2 \mathrm{mM}$ PA

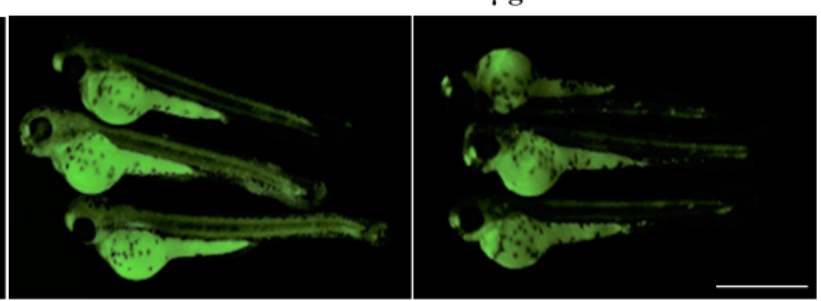

d

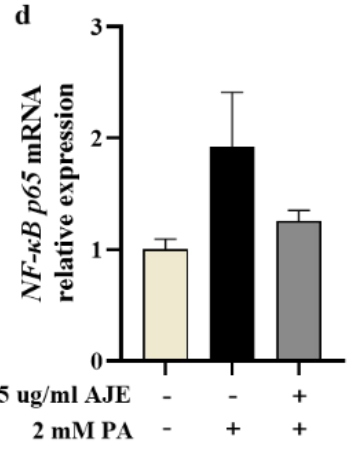

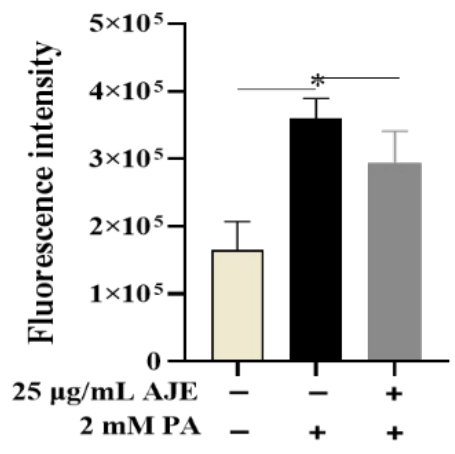

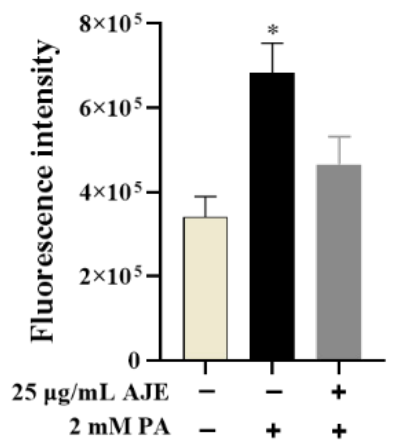

Fig. 2. Inhibitory effect of Apostichopus japonicus extract (AJE) on palmitate (PA)-induced nitric oxide (NO) and cell death in zebrafish larvae. $86 \mathrm{hpf}$ larvae were incubated with diaminofluorophore 4-amino-5-methylamino-2,7'-difluorofluorescein diacetate (DAF-FM DA, $5 \mathrm{mM}$ ) for $30 \mathrm{~min}$ and acridine orange $(\mathrm{AO}, 5 \mu \mathrm{g} / \mathrm{mL}$ ) for $15 \mathrm{~min}$ to detect NO production $(\mathrm{a}, \mathrm{c})$ and cell death $(\mathrm{b}, \mathrm{d})$, respectively. The experiments were performed in triplicate. $86 \mathrm{hpf}$ embryos were stained with $5 \mu M$ DAF-FM-DA (a) or $5 \mu \mathrm{g} / \mathrm{mL} \mathrm{AO}$ (e) for 30 min at $28.5^{\circ} \mathrm{C}$ in the dark. Scale bar: $1 \mathrm{~mm} .{ }^{*} p<0.05,{ }^{* *} p<0.01$ and ${ }^{* * * *} p<0.001$. hpf, hours post fertilization.

attacks and strokes, thereby reducing premature morbidity, mortality and disability (WHO, 2007). Evidence confirming that A. japonicus has antioxidant, hypolipidemic, antifungal, anticoagulant and anti-skin cancer activities (Kim et al., 2017; Liu et al., 2012; Wang et al.; 2012, Yang et al., 2015a; Yang et al., 2015b). To our knowledge, its protective effect on blood vessels is not reported so far. PA is well known to damage the proliferation and invasion of HUVECs in vitro, and increase cell apoptosis (WHO, 2007). Therefore, this study firstly established the PA-induced apoptosis model of HUVECs, and confirmed that AJE has protective effect on PA-induced damage in HUVECs (Data was not shown). Furthermore, we established an in vivo 
a
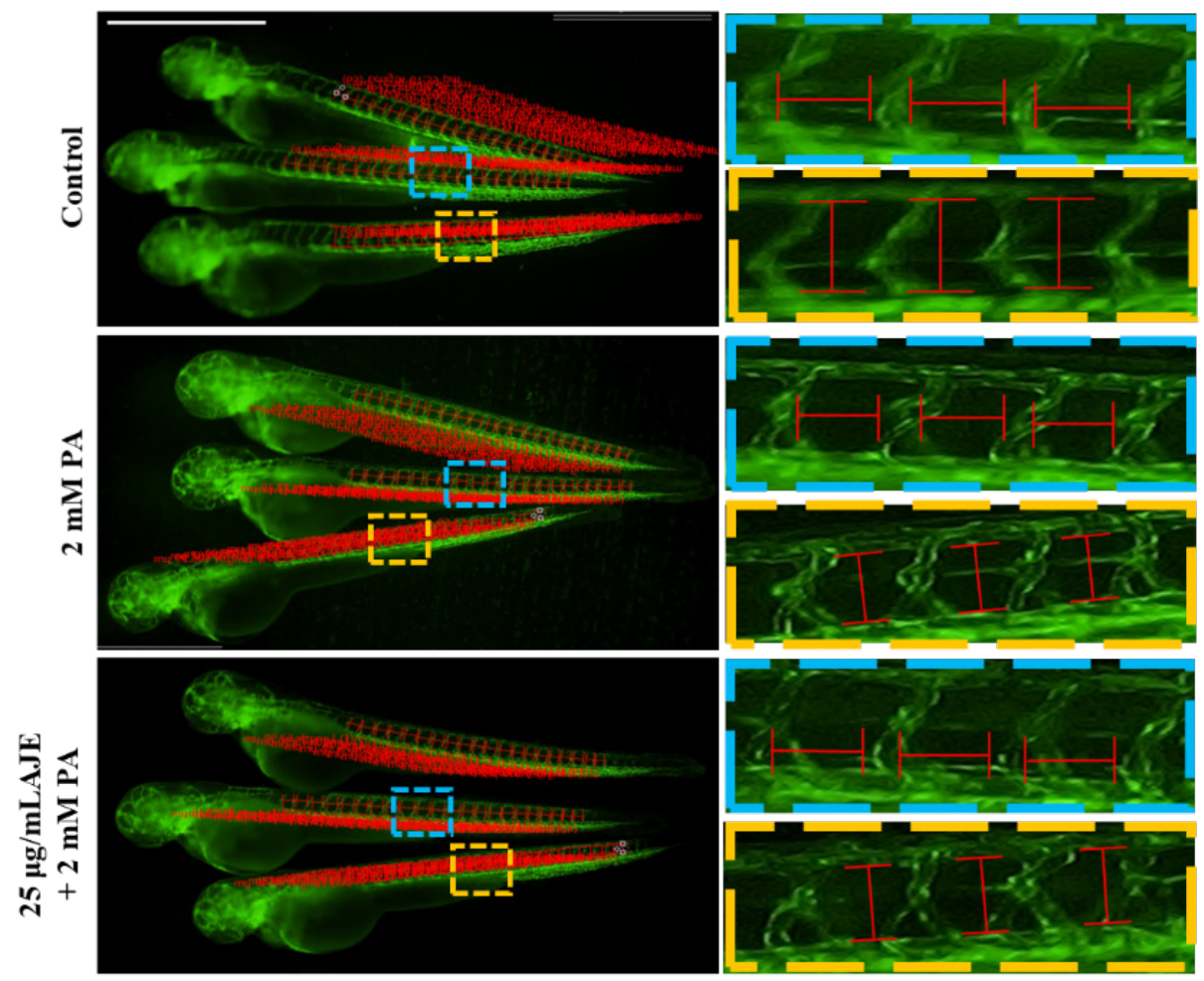

c

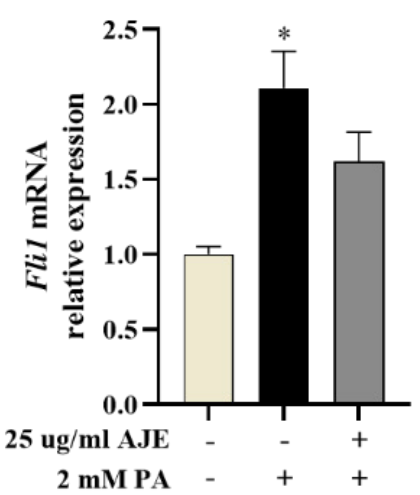

d

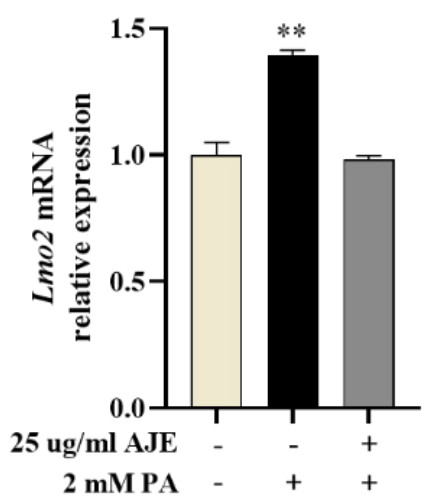

b
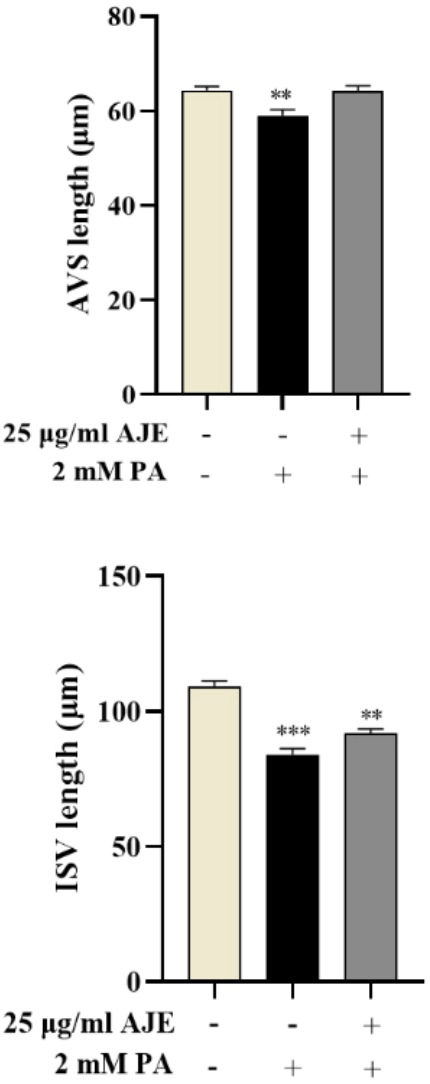

Fig. 3. Apostichopus japonicus extract (AJE) attenuated palmitate (PA)-induced toxicity on vascular morphogenesis in $\mathrm{Tg}$ (fli1a:eGFP) transgenic zebrafish. (a) Fluorescence images of $T g$ (fli1a:eGFP) transgenic zebrafish embryos at $60 \mathrm{hpf}$ treated with phenylthiourea (PTU, control), PA and pretreatment of AJE with PA. The red horizontal and vertical rows in blue and yellow dotted boxes represent the partial view of axial vascular segment (AVS) and intersegmental vessel (ISV) length, respectively. (b) The length of AVS and ISV were measured by cellSens software. The relative mRNA expression of (c) Fli1, and (d) Lmo2 were analyzed by qPCR. All of measurements were performed in triplicate. Scale bar: $1 \mathrm{~mm} .{ }^{*} p<0.05,{ }^{* *} p<0.01$ and ${ }^{* * * *} p<0.001$. hpf, hours post fertilization.

zebrafish model induced by PA and evaluated the efficacy of AJE-pretreatment. In this study, AJE-pretreatment reduced the physiological indicators similar to the symptoms of CVDs, such as mortality, accelerated heartbeats and malformations induced by PA in zebrafish embryos (Fig. 1). These results preliminarily suggested that AJE may have the potential to prevent CVDs due 
to its inhibitory effect on PA-induced toxicity in both in vitro and in vivo.

Previous studies demonstrated that abnormal production or activity of $\mathrm{NO}$ is considered to be a major mechanism of CHD as a CVD (Besedina, 2016). As well as iNOS-derived NO is also proposed as adverse effects on myocardium (D'Oria et al., 2020). Evidence confirmed that increased expression of iNOS was detected in the endothelium of coronary arteries in aged rats (Csiszar et al., 2002; Santhanam et al., 2007). Additionally, the upregulation and overexpression of iNOS can induce cardiac apoptosis, hypertrophy, dilatation and fibrosis in mice (Umar \& van der Laarse, 2010). In the present study, PA-induced excessive NO production and iNOS mRNA expression in zebrafish which was downregulated by AJE-pretreatment compared with the control (Fig. 1a, b). This result could explain the phenomenon of accelerated heartbeat may cause by the detrimental effects of iNOS-derived NO on zebrafish myocardium. Furthermore, evidence shows that diverse processes induced CVDs are converging on regulation of TNF- $\alpha$ signaling and result in endothelial dysfunction and vascular disease. TNF- $\alpha$ can independently or depend on the activation of transcription factors, such as NF- $\mathrm{kB}$, to induce gene expressions of various inflammatory cytokines and chemokines. This eventually leads to vascular remodeling, inflammation and oxidative stress, as well as atherosclerosis, thrombosis, endothelial cell apoptosis and impaired NO bioavailability (Zhang et al., 2009). In this study, mRNA levels of TNF- $\alpha$ and NF- $\mathrm{kB}$ were elevated by PA treatment while largely reduced after pretreatment of AJE (Fig. 1c, d). In addition, PA-induced embryonic cell death was also ameliorated by AJE-pretreatment (Fig. 1e). These results suggest that AJE probably block the NF-kB signaling pathway by inhibiting the overexpression of TNF- $\alpha$, and ultimately prevent cell death induced by PA in zebrafish embryos.

Fli1 gene participates in the regulatory process of vasculogenesis and angiogenesis. Fli1 deficient xenopus and zebrafish embryos display a block in the development of hemangioblast, whereas Fli1 overexpression was observed in a variety of cancers and diseases (Li et al., 2015). In addition, Fli1 induces the expression of key hemangioblast genes such as Lmo2 (Liu et al., 2008). Lmo2 is a crucial determinant of tissue healing and angiogenesis in vivo zebrafish. Meng et al. identified that gene expression of Lmo2 elevated during caudal fin resection and regeneration (Meng et al., 2016). Further studies demonstrated that Lmo2 knockdown decreased the length and number of ISVs, impaired axial vessel formation and migration of endo- thelial cells (Matrone et al., 2017; Patterson et al., 2005). On the other hand, overexpression of Lmo2 caused T-cell lymphoblastic leukemia in mouse thymus (McCormack et al., 2010). In the present study, the length of AVS and IVS in Tg (fli1a:eGFP) zebrafish embryos was reduced under PA stimulation, while AJE-pretreatment ameliorated it close to the control (Fig. 2a, b). Furthermore, AJE-pretreatment protected against PA-induced overexpression of Fli1 and Lmo2 and maintained it at the control level (Fig. 2c, d). As we know, PA could inhibit angiogenesis by interfering with endothelial cell function (Zhang et al., 2017). In this study, we observed that PA-induced a mild or severe antiangiogenic phenotype on $\mathrm{Tg}$ (fli1a:eGFP) lines (data not shown). Therefore, our study suggests that PA probably induce varying degrees of embryonic anti-angiogenesis and trigger the up-regulation of Fli1 and Lmo2 expression levels to compensate for the dysfunction or damage of endothelial cells caused by PA, while AJE-pretreatment can block the negative effects of PA on zebrafish embryos.

Anti-TNF therapy is widely used in the treatment of many diseases including CVDs (Monaco et al., 2015). However, recent study pointed out that TNF inhibitors could decrease the CVDs events via controlling systemic inflammation, on the contrary can increase the risk of overall and certain cancers (Lee et al., 2018; Yuan et al., 2020). Our results suggest AJE, on the one hand, could be used as a substitute for TNF inhibitor to prevent CVDs by decreasing PA-induced over expression of TNF- $\alpha$; on the other hand, may have the potential to reduce cancer risk by protecting zebrafish embryos against PA-induced overexpression of Fli1 and Lmo2. However, further study is necessary to elucidate.

In conclusion, AJE exhibited vascular protective effect in vivo zebrafish embryo models, AJE protected against PA-induced embryotoxicity by maintaining normal physiological indicators, regulating inflammation and genes expression related to vascular development. These findings might provide a potential mechanism basis for AJE in the prevention of CVDs.

\section{Competing interests}

No potential conflict of interest relevant to this article was reported.

\section{Funding sources}

Not applicable. 


\section{Acknowledgements}

This research supported by research supporting program of the Hanseo University in 2021.

\section{Availability of data and materials}

Not applicable.

\section{Ethics approval and consent to participate}

This article does not require IRB/IACUC approval because there are no human and animal participants.

\section{ORCID}

Chunying Zhang

https://orcid.org/0000-0001-8647-1409

Seon-Heui Cha

https://orcid.org/0000-0002-5004-3223

\section{References}

Aires RD, Capettini LSA, Silva JF, Rodrigues-Machado MG, Pinho V, Teixeira MM, et al. Paraquat poisoning induces TNF- $\alpha$-dependent iNOS/NO mediated hyporesponsiveness of the aorta to vasoconstrictors in rats. PLOS ONE. 2013;8:e73562.

Besedina A. NO-synthase activity in patients with coronary heart disease associated with hypertension of different age groups. J Med Biochem. 2016;35:43-9.

Carter AM. Complement activation: an emerging player in the pathogenesis of cardiovascular disease. Scientifica. 2012;2012:402783.

Chen S, Hu Y, Ye X, Li G, Yu G, Xue C, et al. Sequence determination and anticoagulant and antithrombotic activities of a novel sulfated fucan isolated from the sea cucumber Isostichopus badionotus. Biochim Biophys Acta Gen Subj. 2012;1820:989-1000.

Csiszar A, Ungvari Z, Edwards JG, Kaminski P, Wolin MS, Koller A, et al. Aging-induced phenotypic changes and oxidative stress impair coronary arteriolar function. Circ Res. 2002;90:1159-66.

D’Oria R, Schipani R, Leonardini A, Natalicchio A, Perrini S, Cignarelli A, et al. The role of oxidative stress in cardiac disease: from physiological response to injury factor. Oxid Med Cell Longev. 2020;2020:5732956.

Ebbesson SOE, Voruganti VS, Higgins PB, Fabsitz RR, Ebbesson LO, Laston S, et al. Fatty acids linked to cardiovascular mortality are associated with risk factors. Int J Circumpolar Health. 2015;74:28055.
Ganta VC, Annex BH. LMO2 (LIM domain only 2) and endothelial cell migration in developmental and postnatal angiogenesis. Arterioscler Thromb Vasc Biol. 2017;37:1806-8.

Giudicessi JR, Ackerman MJ, Camilleri M. Cardiovascular safety of prokinetic agents: a focus on drug-induced arrhythmias. Neurogastroenterol Motil. 2018;30:e13302.

Hossain A, Dave D, Shahidi F. Northern sea cucumber ( $\mathrm{Cu}$ cumaria frondosa): a potential candidate for functional food, nutraceutical, and pharmaceutical sector. Mar Drugs. 2020;18:274.

Hu S, Wang J, Li Z, Fu J, Wang Y, Xue C. Hpyerglycemic effect of a mixture of sea cucumber and Cordyceps sinensis in streptozotocin-induced diabetic rat. J Ocean Univ China. 2013;13:271-7.

Kaptoge S, Pennells L, De Bacquer D, Cooney MT, Kavousi M, Stevens G, et al. World Health Organization cardiovascular disease risk charts: revised models to estimate risk in 21 global regions. Lancet Glob Health. 2019;7:E1332-45.

Kareh M, El Nahas R, Al-Aaraj L, Al-Ghadban S, Naser Al Deen $\mathrm{N}$, Saliba N, et al. Anti-proliferative and anti-inflammatory activities of the sea cucumber Holothuria polii aqueous extract. SAGE Open Med. 2018;6:2050312118809541.

Kim NY, Choi WY, Heo SJ, Kang DH, Lee HY. Anti-skin cancer activities of Apostichopus japonicus extracts from low-temperature ultrasonification process. J Healthc Eng. 2017;2017:6504890.

Lawson ND, Weinstein BM. In vivo imaging of embryonic vascular development using transgenic zebrafish. Dev Biol. 2002;248:307-18.

Lee JL, Sinnathurai P, Buchbinder R, Hill C, Lassere M, March L. Biologics and cardiovascular events in inflammatory arthritis: a prospective national cohort study. Arthritis Res Ther. 2018;20:171.

Li S, Dang YY, Oi Lam Che G, Kwan YW, Chan SW, Leung GPH, et al. VEGFR tyrosine kinase inhibitor II (VRI) induced vascular insufficiency in zebrafish as a model for studying vascular toxicity and vascular preservation. Toxicol Appl Pharmacol. 2014;280:408-20.

Li Y, Luo H, Liu T, Zacksenhaus E, Ben-David Y. The ets transcription factor Fli-1 in development, cancer and disease. Oncogene. 2015;34:2022-31.

Liu F, Walmsley M, Rodaway A, Patient R. Fli1 acts at the top of the transcriptional network driving blood and endothelial development. Curr Biol. 2008;18:P1234-40.

Liu H, Wang H. Early detection system of vascular dis- 
ease and its application prospect. BioMed Res Int. 2016;2016:1723485.

Liu HH, Ko WC, Hu ML. Hypolipidemic effect of glycosaminoglycans from the sea cucumber Metriatyla scabra in rats fed a cholesterol-supplemented diet. J Agric Food Chem. 2002;50:3602-6.

Liu X, Sun Z, Zhang M, Meng X, Xia X, Yuan W, et al. Antioxidant and antihyperlipidemic activities of polysaccharides from sea cucumber Apostichopus japonicus. Carbohydr Polym. 2012;90:1664-70.

Mansour MB, Balti R, Yacoubi L, Ollivier V, Chaubet F, Maaroufi RM. Primary structure and anticoagulant activity of fucoidan from the sea cucumber Holothuria polii. Int J Biol Macromol. 2019;121:1145-53.

Matrone G, Meng S, Gu Q, Lv J, Fang L, Chen K, et al. Lmo2 (LIM-domain-only 2) modulates Sphk1 (sphingosine kinase) and promotes endothelial cell migration. Arterioscler Thromb Vasc Biol. 2017;37:1860-8.

McCormack MP, Young LF, Vasudevan S, De Graaf CA, Codrington R, Rabbitts TH, et al. The Lmo2 oncogene initiates leukemia in mice by inducing thymocyte self-renewal. Science. 2010;327:879-83.

Meng S, Matrone G, Lv J, Chen K, Wong WT, Cooke JP. LIM domain only 2 regulates endothelial proliferation, angiogenesis, and tissue regeneration. J Am Heart Assoc. 2016;5:e004117.

Miller V, Mente A, Dehghan M, Rangarajan S, Zhang X, Swaminathan S., et al. Fruit, vegetable, and legume intake, and cardiovascular disease and deaths in 18 countries (PURE): a prospective cohort study. Lancet. 2017;390:2037-49.

Monaco C, Nanchahal J, Taylor P, Feldmann M. Anti-TNF therapy: past, present and future. Int Immunol. 2015;27:55-62.

Patan S. Vasculogenesis and angiogenesis as mechanisms of vascular network formation, growth and remodeling. J Neurooncol. 2000;50:1-15.

Patterson LJ, Gering M, Patient R. Scl is required for dorsal aorta as well as blood formation in zebrafish embryos. Blood. 2005;105:3502-11.

Santhanam L, Lim HK, Lim HK, Miriel V, Brown T, Patel M., et al. Inducible NO synthase-dependent S-nitrosylation and activation of arginase 1 contribute to age-related endothelial dysfunction. Circ Res. 2007;101:692-702.

Sarmah S, Marrs JA. Zebrafish as a vertebrate model system to evaluate effects of environmental toxicants on cardiac development and function. Int J Mol Sci. 2016;17:2123.
Seo D, Ginsburg GS, Goldschmidt-Clermont PJ. Gene expression analysis of cardiovascular diseases: novel insights into biology and clinical applications. J Am Coll Cardiol. 2006;48:227-35.

Sinha S, Perdomo G, Brown NF, O'Doherty RM. Fatty acid-induced insulin resistance in L6 myotubes is prevented by inhibition of activation and nuclear localization of nuclear factor kappa B. J Biol Chem. 2004;279:41294-301.

Stoclet JC, Muller B, Andriantsitohaina R, Kleschyov A. Overproduction of nitric oxide in pathophysiology of blood vessels. Biochemistry (Mosc). 1998;63:826-32.

Sun HJ, Wu ZY, Nie XW, Bian JS. Role of endothelial dysfunction in cardiovascular diseases: the link between inflammation and hydrogen sulfide. Front Pharmacol. 2020;10:1568.

Tang GY, Meng X, Li Y, Zhao CN, Liu Q, Li HB. Effects of vegetables on cardiovascular diseases and related mechanisms. Nutrients. 2017;9:857.

Thomé MP, Filippi-Chiela EC, Villodre ES, Migliavaca CB, Onzi $\mathrm{GR}$, Felipe KB, et al. Ratiometric analysis of acridine orange staining in the study of acidic organelles and autophagy. J Cell Sci. 2016;129:4622-32.

Tufail T, Saeed F, Abbas M, Arshad MU, Nadeem MT, Bader ul Ain H, et al. Marine bioactives: potentials to reduce the incidence of cardiovascular disorders. Curr Top Nutraceutical Res. 2018;16.

Umar S, van der Laarse A. Nitric oxide and nitric oxide synthase isoforms in the normal, hypertrophic, and failing heart. Mol Cell Biochem. 2010;333:191-201.

Volpe CMO, Abreu LFM, Gomes PS, Gonzaga RM, Veloso CA, Nogueira-Machado JA. The production of nitric oxide, IL6, and TNF-alpha in palmitate-stimulated PBMNCs is enhanced through hyperglycemia in diabetes. Oxid Med Cell Longev. 2014;2014:479587.

Wang Z, Zhang H, Yuan W, Gong W, Tang H, Liu B, et al. Antifungal nortriterpene and triterpene glycosides from the sea cucumber Apostichopus japonicus Selenka. Food Chem. 2012;132:295-300.

World Health Organization [WHO]. Prevention of cardiovascular disease: guidelines for assessment and management of total cardiovascular risk. Geneva: World Health Organization; 2007.

Yang J, Wang Y, Jiang T, Lv L, Zhang B, Lv Z. Depolymerized glycosaminoglycan and its anticoagulant activities from sea cucumber Apostichopus japonicus. Int J Biol Macromol. 2015a;72:699-705. 
Yang J, Wang Y, Jiang T, Lv Z. Novel branch patterns and anticoagulant activity of glycosaminoglycan from sea cucumber Apostichopus japonicus. Int J Biol Macromol. 2015b;72:9118.

Yuan S, Carter P, Bruzelius M, Vithayathil M, Kar S, Mason AM, et al. Effects of tumour necrosis factor on cardiovascular disease and cancer: a two-sample Mendelian randomization study. eBioMedicine. 2020;59:102956.

Zhang H, Hao J, Sun X, Zhang Y, Wei Q. Circulating pro-angiogenic micro-ribonucleic acid in patients with coronary heart disease. Interact Cardiovasc Thorac Surg. 2018;27:336-42.

Zhang H, Park Y, Wu J, Chen X, Lee S, Yang J, et al. Role of TNF- $\alpha$ in vascular dysfunction. Clin Sci. 2009;116:219-30.

Zhang J, Shan Y, Li Y, Luo X, Shi H. Palmitate impairs angiogenesis via suppression of cathepsin activity. Mol Med Rep. 2017;15:3644-50.

Zhi Y, Lu H, Duan Y, Sun W, Guan G, Dong Q, et al. Involvement of the nuclear factor- $\kappa \mathrm{B}$ signaling pathway in the regulation of CXC chemokine receptor- 4 expression in neuroblastoma cells induced by tumor necrosis factor- $\alpha$. Int J Mol Med. 2015;35:349-57.

Zhou DD, Luo M, Shang A, Mao QQ, Li BY, Gan RY, et al. Antioxidant food components for the prevention and treatment of cardiovascular diseases: effects, mechanisms, and clinical studies. Oxid Med Cell Longev. 2021;2021:6627355.

Zhu H, Traver D, Davidson AJ, Dibiase A, Thisse C, Thisse B, et al. Regulation of the $1 m o 2$ promoter during hematopoietic and vascular development in zebrafish. Dev Biol. 2005;281:256-69.

Ziff OJ, Kotecha D. Digoxin: the good and the bad. Trends Cardiovasc Med. 2016;26:585-95. 